\title{
Characterization of Materials at the Nanoscale Using Hard X-ray Microspectroscopy Techniques.
}

\author{
$\underline{\text { Gema Martínez-Criado }}^{1,2,}{ }^{*}$, Rémi Tucoulou ${ }^{2}$, Julie Villanova $^{2}$, Damien Salomon $^{2}$ and Sylvain Labouré ${ }^{2}$ \\ 1. Instituto de Ciencia de Materiales de Madrid (CSIC), 28049-Cantoblanco, Madrid, Spain \\ 2. Experiments Division, European Synchrotron Radiation Facility, 38043-Grenoble, France \\ * Corresponding author, gema.martinez.criado@csic.es
}

Hard X-ray spectromicroscopy techniques are key tools with relevant applications across multiple fields. Here we briefly describe how these tools are implemented and used nowadays for the characterization of heterogeneous materials at the nanoscale. We shortly present the essential role of the associated instrumentation for reliable 2D and 3D data acquisitions with nanometer spatial resolution. Two examples exemplify the potential of hard X-ray modalities to provide new insights into hybrid perovskites for solar cells and nanowires for light emitting diodes.

Today the ability to resolve spatially elemental, chemical, optical and structural features at the nanoscales with high sensitivity (e.g., sub-ppb level) is fundamental to explain the macroscopic behaviors of heterogeneous materials. Ferroelectric domains, nanoporous compounds, nanoparticles, diluted and/or embedded nanostructures in amorphous matters are just a few examples in which ultrafine elements moderate average physical properties. Therefore, in materials science the application of spatially resolved X-ray methods represents a pivotal step in the investigation of local transformations influenced by small heterogeneities, surfaces, grain boundaries, interfaces, etc. According to the rich and multiple radiation-matter interactions, hard X-ray nanobeams have been lately exploited in several stateof-the-art approaches [1]. By means of nanofocusing optics, usually a hard X-ray beam is focused to a very small spot size, ranging from hundreds to tens of nanometers. The sample is raster scanned through the beam whereas the intensity of different signals is recorded in a pixel-by-pixel basis. Based on the images generated by a contrast mechanism (e.g., X-ray fluorescence (XRF)), spatially resolved microspectroscopy data can be collected across the sample at individual points to extract information on elemental composition, valence states, symmetry, electronic states, coordination number, interatomic distances, etc. Alternatively, by recording images at different hard X-ray photon energies, for example, full spectral information collected at every pixel can be used to generate spatially resolved information in 2D. As a result, microspectroscopy and spectromicroscopy are often terms applied to spatiallyresolved hard X-ray tools [2].

High brilliant and low-emittance synchrotron facilities (diffraction-limited storage rings), forefront nanofocusing devices, superior detection systems, and novel modalities have played a key role in the hard X-ray microspectroscopy evolution. Complementing soft X-ray related tools, the combination of large penetration depths, high throughput and large tunability in terms of probe (energy, spot size, photon flux, time structure, polarization, etc.) with in-situ or operando exploration has been also an unprecedented asset at nanoscales [3]. However, these advances have proven difficult to technically combine a fixed focal spot position, chromaticity of the focusing lens, angular stability of the double crystal monochromator, fast energy scanning speeds, incident beam intensity monitoring, vibration-free operation, large k-space information and low radiation dose.

Thus, several important technical points should be seriously taken into account to routinely guarantee a 
vibration-free and stable design. The high heat load typically produced by insertion devices operated in hard X-ray micro-spectroscopy beamlines requires optimization of the cooling systems, positions and quality of all optical elements. The large source size demagnifications of long length beamlines (100 200 meters) calls for an extreme stable fixed-exit double crystal monochromator with cutting edge capabilities (e.g., extreme angular stability ( $<<1 \mu \mathrm{rad}$ ), repeatability, thermal, etc.) [4]. Regarding the nanofocusing optics, many factors can influence the focusing properties like thermal distortions, roughness (e.g., superpolished $<1 \AA)$, fabrication slope errors $(\sim 0.1 \mu \mathrm{rad} \mathrm{rms})$ and figure errors $(\sim 1 \mathrm{~nm}$ peak to valley). Sample piezo-stages with very high thermal/vibration stability, resolution, repeatability, low angular errors and ultra-high precision are critical. Thus, online metrology is becoming crucial to reach ultra-high precision operation (errors compensation using laser interferometers/capacitive sensors to measure online angular errors and introduce online compensation, vibration control techniques, etc). Superior detection systems and cryogenic techniques to reduce radiation damage are also keystones.

The following paragraphs describe two recent examples of research highlights focused mainly on, but certainly not limited to, the following research areas: hybrid perovskites for solar cells and nanowires for light emitting diodes.

The first example deals with highly cost-effective optoelectronic materials: the so-called hybrid perovskites [5]. They play an essential role in the modern world by converting an electric current to light (e.g., LED), or conversely by capturing light to produce electricity (e.g., solar cells). Although hybrid perovskites has significant potential, in practice there are still several challenges, including rapid modifications in output in response to a voltage. Previous experiments were unable to directly observe the chemical origin of these changes. At Advanced Photon Source, using operando hard XRF nanospectroscopy researchers have for the first time directly measured nanoscale changes in the chemical composition of crystals of methylammonium lead bromide, a leading hybrid perovskite candidate for optoelectronics [5]. These compounds exhibit exceptional optoelectronic characteristics with solar conversion efficiencies of over $22 \%$ demonstrated in the lab. A highly collimated X-ray nanobeam $(250 \mathrm{~nm}$ spot size with $10^{9} \mathrm{ph} / \mathrm{sec}$ ) mapped each sample's heavier elements (bromine and lead) at $13.6 \mathrm{keV}$ with a parts-per-million sensitivity under an applied electric field. Following the XRF measurements, a laser sequentially probed the same microscopic area to determine its optoelectronic response via photoluminescence (PL). The experiments revealed how the presence of an applied voltage depleted the bromine $(\mathrm{Br})$ within regions of the material, degrading its optoelectronic performance. A higher local bromide concentration was correlated to

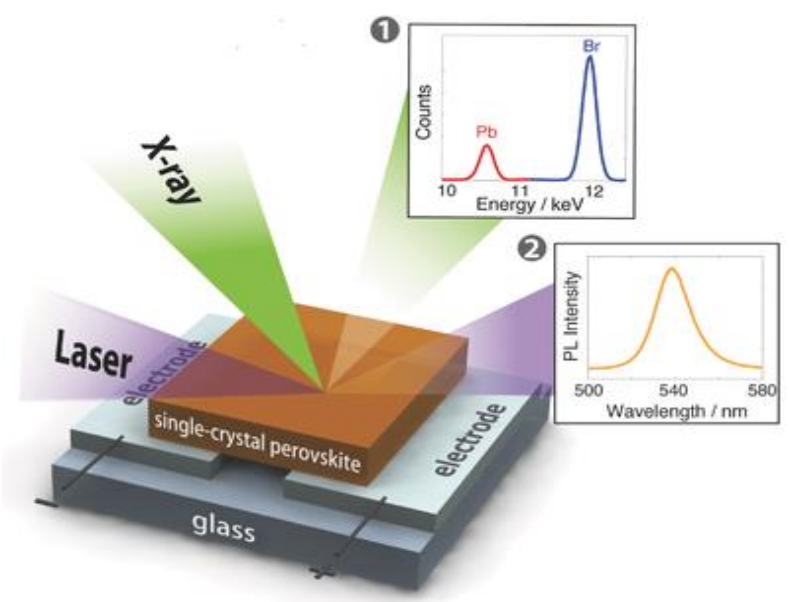

Figure. 1. Photon-in photon-out correlative microscopy: schematic of XRF nanoprobe and spatially resolved PL. Credit: Y. Luo et al. superior optoelectronic performance in $\mathrm{CH}_{3} \mathrm{NH}_{3} \mathrm{PbBr}_{3}$. A lower limit on the electromigration rate was calculated from these experiments and the motion interpreted as vacancy-mediated migration based on theoretical simulations. The 2D XRF data provided direct evidence of field-assisted ionic migration in a model hybrid-perovskite thin single crystal, while the link with PL proved that the halide stoichiometry plays a key role in the optoelectronic properties of the perovskite [5]. 
The second example actually exploits a single hard X-ray nanobeam to simultaneously probe both the XRF and X-ray excited optical luminescence (XEOL); thus, both optical and chemical information are concurrently correlated [6]. With a $70 \times 55 \mathrm{~nm}^{2}$ beam size, the researchers applied such experimental scheme developed at the beamline ID16B of the European Synchrotron Radiation Facility to 1D GaN structures. GaN material, the 2014 Nobel Prize in Physics, is the main semiconductor used for the fabrication of visible light emitting diodes. But, the traditional LED structure suffers from a high number of defects induced by the lattice mismatch and internal electric fields along the $c$ axis. GaN nanowires alternatively present great potential to enhance the performance of these LEDs due to their large surface to volume ratio and nonpolar facets. However, the characterization of their structural, electrical and optical properties, which are highly dependent on the growth conditions, was a challenge at the nanoscale. Salomon and co-workers utilized a high energy nanobeam $(29.6 \mathrm{keV})$ at ID16B to reveal experimentally the strong influence of silane injection during growth on the material quality [6]. Their work provided strong evidence of the role of silane to promote the N-polarity, light emission, and elemental incorporation within single wires. The N-polar material was found to be energetically preferred in the presence of $\mathrm{Si}$, likely preventing the formation of the yellow band related defects. These findings were corroborated from the top surface of a wire covered coaxially by five times $\mathrm{InGaN} / \mathrm{GaN}$ multiquantum wells. A nonuniform indium deposition, accompanied by morphological defects, facets with further $\mathrm{Si}$ doping modulation, and mixed polarity, was shown at the top of the radial structure. In addition, the experiment demonstrated the ability to spatially examine carrier diffusion phenomena without electrical contacts from the radiative recombination from
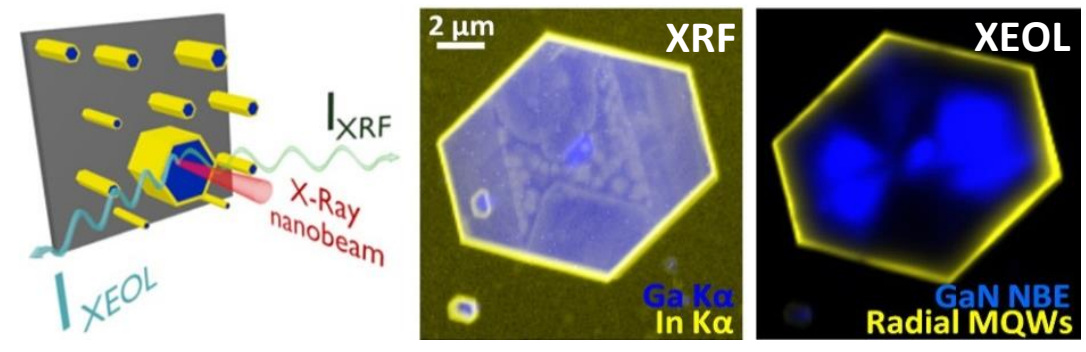

Figure. 2. Schematic of the experimental setup and examples of XRF and XEOL maps collected simultaneously on standing InGaN/GaN core-shell radial wires in yellow and blue. Credit: D. Salomon et al. highly localized silane induced N-polar domains, as well as from InGaN/GaN quantum wells [6]. The enterprising use of chemically heterogeneous nanostructures with dual polarity was actually proposed for future innovation in light emitters of multiple wavelengths even when using defective wires.

\section{References:}

[1] G E Rice et al, Science 334 (2011) 1234.

[2] G Martinez-Criado in "Chapter: Application of Micro and Nanobeams for Materials Science" in book: Synchrotron Light Sources and Free-Electron Lasers, eds. E. Jaeschke, S. Khan, J.R. Schneider, J.B. Hastings, (Springer International Publishing) p. 1505.

[3] L Mino et al, Reviews of Modern Physics (2018) in press.

[4] R Tucoulou et al, J. Synchrotron Rad. 15 (2008) 392.

[5] Y Luo et al, Advanced Materials 29 (2017) 1703451.

[6] D Salomon et al, Nano Letters 17 (2017) 946.

[7] H Yoneda et al, Nature Communications 5 (2014) 5080.

G. Martínez-Criado has been partially supported by the ESRF, CSIC \& MINECO (EUIN-2017-88844). 
https://doi.org/10.1017/S1431927618014836 Published online by Cambridge University Press 